A F C R L - $62-1125$

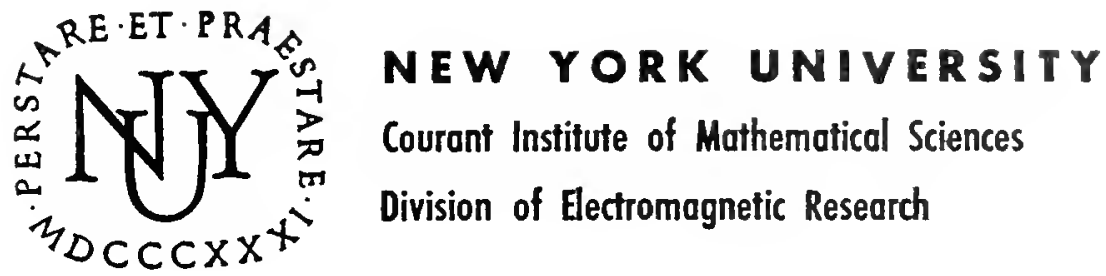

RESEARCH REPORT NO. MH-I3

NEW YORK UNIVERSITY

COURANT INSTITUTE - LIBRARY

4 Washington Place, New York 3, N. Y.

\title{
Analysis of the Transition Region Between A Uniform Plasma and its Confining Magnetic Field: II
}

JAMES HURLEY

\footnotetext{
Contract No.AF $19(604) 6144$

DECEMBER， 1962
} 
, 
ANALYSIS OF THE TRANSITION REGION BETWEEN A UNIFORM PLASMA AND ITS CONFINING MAGNETIC FIEID: II

$$
\text { James Hurley }
$$

Research Report No. MH - 13

\author{
New York University
}

Courant Institute of Mathematical Sciences

Division of Electromagnetic Research

\author{
Contract No. AF $19(604) 6144$ \\ Project 8600 \\ Task 86004
}

December, 1962

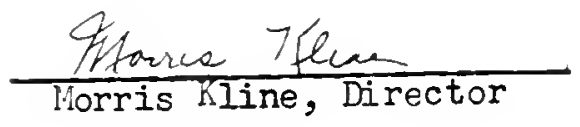

Prepared for:

Geophysics Research Directorate

Air Force Cambridge Hesearch Laboratories

Office of Aerospace Research

United States Air Force

Bedford, Massachusetts

Reproduction in whole or in part is permitted for any purpose of the U.S. Government 
Requests for additional copies by Agencies of the Lepartment of Iefense, their contractors, and other Government agencies should be directed to the:

\author{
ARIED SERVICES TECHIICAL INFORIATION AGENCY \\ ARLINGTON HALL STATION \\ ARLINGTON 12, VIRGINIA
}

Lepartment of Defense contractors must be established for ASTIA services or have their 'need-to-know' certified by the cognizant military agency of their project or contract.

All other persons and organizations should apply to the:

U.S. DEPARTMENT OF COMIERCE OFFICE OF TECHNICAL SERVICES WASHINGTON 25, D.C. 
Abstract

The problem considered here is that of the transition region separating a uniform plasma from its confining magnetic field. The geometry chosen is one-dimensional. The magnetic field profile within the boundary layer is detemined by the current distribution i.e., by the paths of the ions and electrons. The paths of these particles on the other hand are detemined by the fields in which they move. An eract, self-consiztent solution of Maxwell's equations and the equations of motion of the particles is obtained. A detailed analysis is made for a hydrogen plasma. These results are then compared with those of a plasma whose positive constituent is the proton and whose negative constituent has a mass which approaches zero and a speed which approaches infinity in such a way that the energy remains finite. The transition layer thickness for these two plasmas agree to one part in $10^{6}$. It is shown that this latter case is equivalent to treating the electrons of the hydrogen plas.a in the guiding center approximation. 



\section{Introduction}

The problem of the transition region between a plasma and its confining magnetic field has been considered by a number of authors ${ }^{\text {-10 }}$. In most cases the emphasis was on "single orbit" systems, i.e., systems in which the orbit of all ions (electrons) of a given energy are identical except for a simple translation. For such systems an approximate solution is possible in that domain where the ion and electron densities are nearly equal. An exact solution can be obtained only by setting the electron temperature equal to ceroll. orr

I I. Bernstein, in "Radiation and Waves in Plasmas", edited by M. Mitchner (Stanford Univerzity Press, Stanford, California, 1961).

2 V.C.A. Ferraro, J. Geophys. Res. 57. 15 (1052).

3 H. Grad. Phys. of Fluids. 4. 1366 (1761).

4 E. G. Harris, II Iuovo Cimento 23, 115 (1262).

5 A. Morozov and I. Solov'ev, JETP, 13, 727 (1061).

6 M. Rosenbluth, in "Magnetohydrodynamics", edited by R. Landshoff (Stanford University Press, Stanford, Califormia, 1957).

7 G. Schmidt and D. Finkelstein, Bull. A.P.S. 6, No. 3, Abstract SAO (1961), to be published.

8 L. Tonks, Phys. Rev. 113, 400 (1959).

9 W. Paskievici, A. Sestero, H. Weitzner, "Boundary Layer in the Confinement of a one Dimensional Plasma", Report No. NYO 9193, Courant Institute of Math. Sci., N.Y.U. (1962).

10 J. Hurley, Phys. Rev., 124, 1307 (1961).

11 An exception is the field reversal problem considered by Harris. 
objective in this paper is to consider a somewhat different physical system; one in which it is possible (and reasonable) to choose the electron density identically equal to the ion density. For this systern we shall be able to erhibit an exact solution without requiring that the electron temperature be zero.

This paper is an extension of an earlier work by the author 10 to include the electron motion. The problem is quite similar to that considered by Tonls ; the essential differences being that we allow the particles a component of velocity in the direction of the magnetic field and that the solution is exact.

Briefly the problem my be described as follows. All quantities are assumed to depend only on a single variable, $x$. The magnetic field is directed everywhere in the z-direction, and is uniform from $x=-\infty$ to $x=0$. As we cross the plane $x=0$ we enter into the plasma domain, where the field decreases and eventually becomes uniform again at $x=+\infty$. The paths of the plasma constituents are detemined by the fields (magnetic and electric) in which they move. The fields are in turn determined by the paths of the particles. Our problem then is to solve, self consistently, Maxwell's equations and the equations of motion of the particles.

These conditions are not sufficient to determine a unique solution. Even if the distribution functions should be specified at infinity there is still considerabl freedom as to how the particles are put into the transition region. This lack of uniqueness allows us to impose a number of additional simplifying restrictions on the solution. The 
most important of these is that of neutrality. Given the ion distribution function (to be prescribed present electron distribution function be such that the electron density is everywhere equal to the ion density. This condition gives the electron distribution function as the solution of an interral equation. We shall also require that all purticles have the same energy and that the distribution functions depend on the - -component of velocity only through the enery. With these rostrictions and a suitable choice for the ion distribution function the solution is unique.

\section{Analysis}

The only one of Marwell's equations that relates to this problem is

$$
\frac{d B}{d x}=-\frac{4 \pi}{c}(i+i)
$$

where $j_{+}$and $j_{\text {- }}$ are the ion and electron currents respectively and are given by

$$
j_{ \pm}= \pm e \int \dot{y} \dot{f}_{ \pm}^{*}(x, \dot{x}, \dot{y}, \dot{z}) d \dot{x} d \dot{y} d \dot{z}
$$

and $f_{+}^{*}$ and $f_{-}^{*}$ are the ion and electron distribution functions. The equations of motion may be integrated once to give three constants of the motion. These are the energy and the generalized momenta in the $\mathrm{y}$ and $\mathrm{z}$ directions 


$$
\begin{aligned}
& \mathrm{E}_{ \pm}= \pm\left(\dot{2}^{2}+\dot{y}^{2}+\dot{2}^{2}\right) \\
& E_{+}=m_{ \pm} \dot{y}+\mathrm{B} / \mathrm{C} \\
& Q_{ \pm}=m_{ \pm} \dot{z}
\end{aligned}
$$

where $A(x)$ is the vector potential defined by

$$
B=d A / d z
$$

and normalised so that

$$
A(0)=0 \text {. }
$$

Since the distribution function must be constant along the trajectories of the particles in phase space (Liouville's equation), the equations of motion of the particles will be satisfied by choosing the distribution function so that it depends only on the constants of motion $E, P$, and $Q^{l}$. Writing $f(E, P, Q)$ for $f^{*}(x, \dot{x}, \dot{y}, \dot{\Xi})$ when $e x-$ pressed as a function of $\mathrm{E}, \mathrm{P}$ and $\mathrm{Q}$ the current may then be written

$$
j_{ \pm}= \pm \frac{2 e}{m_{ \pm}} \int(P \bar{F} e A / c) I_{ \pm}(E, P, Q) \frac{\partial(\dot{x}, \dot{y}, \dot{z})}{\partial(E, P, Q)} d E d P d Q
$$

where $\partial(\dot{x}, \dot{y}, \dot{z}) / \partial(E, P, Q)$ is the Jacobian of the transformation and is

$$
\frac{\partial(x, y, z)}{\partial(E, P, Q)}=\frac{1}{m_{ \pm}^{3} \dot{x}}=1 / m_{ \pm}^{2} \sqrt{2 m_{ \pm} E-\left(P \mp \frac{e A}{c}\right)^{2}-Q^{2}}
$$

1 See for example Ref. 3 and 4. 
A factor of two has been inserted in (4) to take account of the mutiplicity of the mapping $(\dot{x} \rightarrow-\dot{x})$. It is clear that the domain of integration must be limited by the inequality

$$
m_{ \pm}^{2} x^{2}=2 m \pm-\left(P \mp \frac{e A}{c}\right)^{2}-Q^{2} \geq 0
$$

that is, to the domain inside a parabola of revolution whose axis is paraliel to the E-axis and whose apex lies at the point ( $. \pm \mathrm{eA} / \mathrm{c}, 0)$.

Our problem may now be stated analytically. Given $f_{+}(\mathrm{E}, P \cdot Q)$, construct an $f_{-}$satisfying the integral equation $N_{+}(x)=I_{-}(x)$ where

$$
I_{ \pm}=\frac{2}{m_{ \pm}^{2}} \int \frac{I_{ \pm} d E d P d Q}{\sqrt{2 m \pm-\left(P \mp \frac{e A}{c}\right)^{2}-Q^{2}}}
$$

and is the ion (electron) muber density. The domain of integration is again given by (5). The currents may then be determined from (4) as functions of A. Substituting these currents into (1) we obtain $A(x)$ as the solution of an ordinary second order differential equation. We shall take for our aistribution function $f_{t}$ the following expression

$$
f_{+}= \begin{cases}\frac{N_{\infty} m_{+}^{2}}{4 \pi \sqrt{2 m_{+} E_{+}}} \delta\left(E-E_{+}\right), & P \geq \sqrt{2 m_{+} E_{+}} \\ 0, & P<\sqrt{2 m_{+} E_{+}}\end{cases}
$$

Such a choice requires 1) that all ions have the same speed, 2) that the distribution be isotropic and uniform at $\mathrm{x}=\infty$ where the density is $\mathbb{N}_{\infty}$ and 3 ) that there be no ions in the domain $x<0$ or equivalently in the domain $A(x)<0$ since we have chosen $A(0)=0$ and we assume that 
$A(x)$ increases monotonically with is (as may be verified rron the solution). This last condition follows immediately from (5) and (6) and the add tional amplifying assumption that $I_{+}$is independent of Q. In fact we may show that for

$$
\mathbb{N}_{+}=0 . \quad x<0
$$

it is both necessary and sufficient that

$$
f_{+}=0, \quad P<\sqrt{2 m+E_{+}}
$$

To show this we note that since all ions have the same energy we may evaluate $E$ in (5) at $E_{+}$. Thus the domin of integration for the $\mathrm{P}-\mathrm{Q}$ variables is a circle of radius $\sqrt{2 m+E_{+}}$centered at $(\mathrm{eA} / \mathrm{c}, \mathrm{O})$ (see figure (1)), a cross section of the parabola of revolution mentioned in connection with (5). Since we require $N_{+}(x)=0$ for all $x<0$ and since $f_{+}$is nomegative it must be zero within all circles in the P-Q plane whose centers were located at $(\mathrm{eA} / \mathrm{c}, 0)$ with $\mathrm{eA} / \mathrm{c} 0$. But as we are looking for a distribution function which is independent of $Q$ we must therefore set $f_{+}=0$ for all $P<\sqrt{2 m_{+} E_{+}}$. Briefly we may say that if $f_{+}=0$ in the half plane $P<\sqrt{2 m_{+} E_{+}}$then certainly $f_{+}=0$ within the circle $P^{2}+Q^{2}=2 m_{+} E_{+}$. In the first case $f_{+}$ depends only on $P$ and in the second on both $P$ and $Q$.

We now set

$$
f_{-}=\frac{\mathbb{N}_{\infty} m_{-}^{2}}{4 \pi \sqrt{2 m_{-} E_{-}}} g(\bar{P}) \delta\left(E-E_{-}\right)
$$




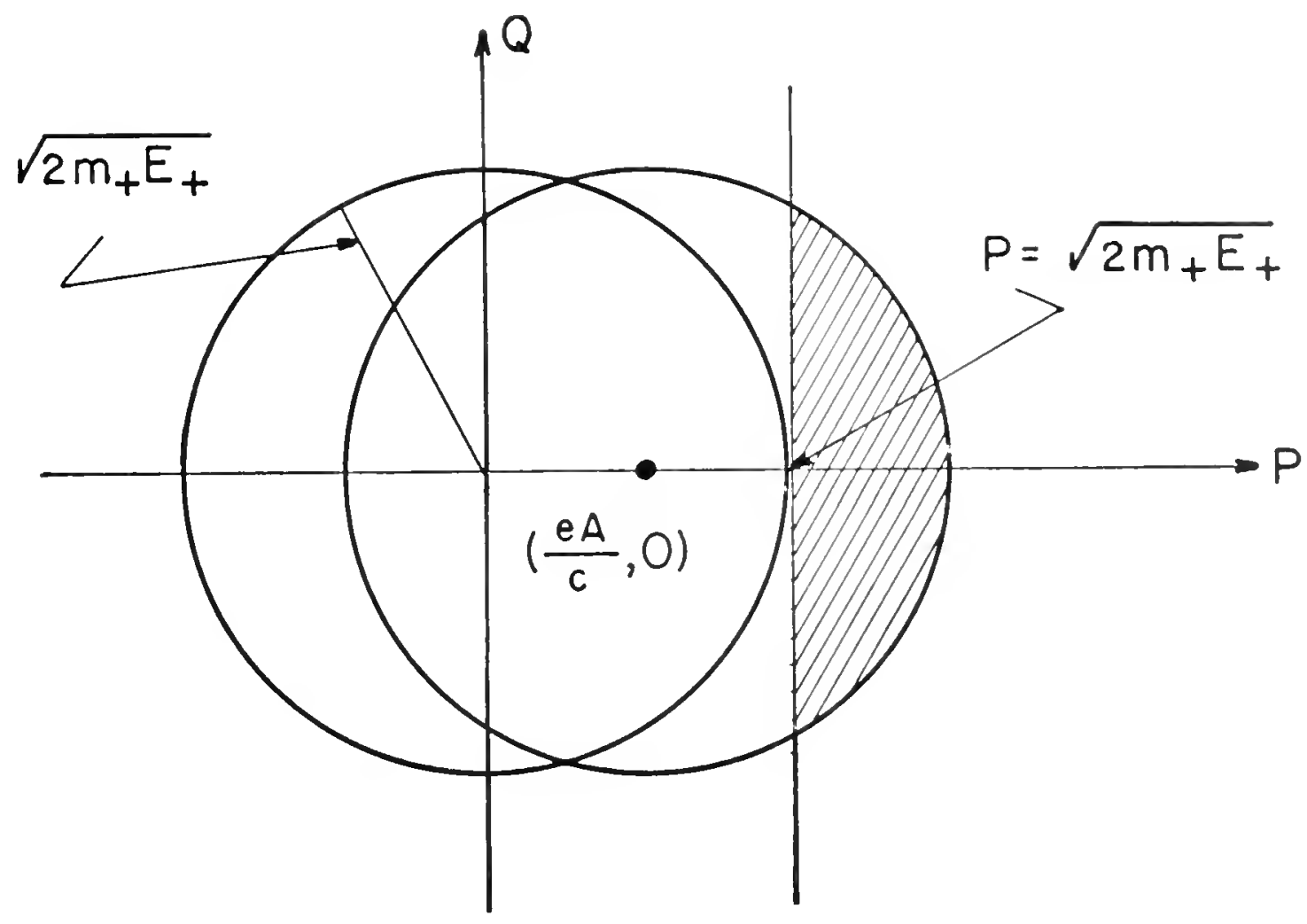

Figure 1. The domain of integration in the $P-Q$ plane is a circle centered at $(\mathrm{eA} / \mathrm{c}, \mathrm{O})$ of radius $\sqrt{2 \mathrm{~m}_{+} \mathrm{E}_{+}}$. The requirement that there be no particles in the domain $x<0$ is satisfied by setting the distribution function equal to zero for all $\mathrm{P}<\sqrt{2 m_{+}{ }^{E}}$ or equivalently by using the shaded area as the effective domain of integration. 
where $g(\bar{P})$ is to be determined by the condition of charce neutrality under the restrictions that $g(\infty)=I$ and $g(\bar{P})=0$ for $\bar{P} \sqrt{2 m-\bar{E}-}$ so that there may be no electrons in the domain $x$. For convenience we have set $\overline{\mathrm{F}}=-\mathrm{P}$. Equating the ion and electron densities given by (6) we have

$$
\mathbb{N}_{ \pm}=\frac{\mathbb{N}_{\infty}}{2 r} \int_{\alpha-r}^{\alpha+r} g(\bar{p}) d \bar{P}=\frac{\mathbb{N}_{\infty}}{2 R}\left\{\begin{array}{cc}
0 & \alpha-0 \\
\alpha & 0 \leq \alpha \leq 2 R \\
2 R & 2 R \leq \alpha
\end{array}\right.
$$

where we have set $\alpha=\mathrm{eA} / \mathrm{c}, r=\sqrt{2 m-E_{-}}$and $\mathrm{R}=\sqrt{2 m_{+} \bar{E}_{+}}$. To solve this equation we differentiate both sides with respect to $\alpha$ and obtain the difference equation

$$
g(\alpha+r)-g(\alpha-r)=\frac{r}{R}\left\{\begin{array}{cc}
0 & \alpha-0 \\
1 & 0 \leq \alpha-2 R \\
0 & 2 R
\end{array}\right.
$$

One may see immediately that the solution is given by $g(\bar{P})$ defined in figure (2). At any point $P=\alpha$ the difference between the function $r$ units to the right and $r$ units to the left of $\alpha$ is everywhere equal to $r / R$ unless $\alpha$ lies outside the interval $O$ to $2 R$ in which case it is zero. We also see that for such a solution to exit it is necessary for $\mathrm{R}$ to be an integral multiple of $r$. This result is not unexpected. It states in effect that the electrons will completely shield the ions if the electron orbit fits precisely into the ion orbit without any overlap. 
Now that the distribution functions $f_{+}$and $f_{-}$are lnown we may calculate the current densities from (4). They are

$$
\begin{aligned}
& j_{+}=j_{0} \frac{\alpha}{R}\left(2-\frac{\alpha}{R}\right) \\
& j_{-}=\frac{E_{-}}{E_{+}} j_{0}\left(\frac{z}{2}-n+1\right)\left(\frac{x}{2}+n+I\right)
\end{aligned}
$$

when $\mathrm{n}$ is an odd integer defined by the inequalities

$$
m-r-i-n+r .
$$

In effect it tells which step the vector potential it is on (see figure (2)). Both ion and electron currents are zero for u outside the interval $O$ to $2 R$. The constant $j_{0}\left(=e_{\infty} R / 4 m_{+}\right)$is the maximum ion current. We see that if the electron and ion energies are equal (and from here on we shall assume this to be the case) their current will also be equal (see figure (3)). If these currents are substituted into ( $I$ ) and both sides mutiplied by da we may integrate once to obtain

$$
\begin{gathered}
\frac{1}{2}(d \alpha / d x)^{2}-\frac{1}{2}(d \alpha / d x)_{0}^{2}=-\frac{4 \pi e}{c} \int_{0}^{\alpha}\left(j_{+}+j_{-}\right) d \alpha \\
=j_{0}\left\{R\left[\left(\frac{\alpha}{R}\right)^{2}-\frac{1}{3}\left(\frac{\alpha}{R}\right)^{3}\right]+\left[\frac{2}{3}(n-1) r+(\alpha-n r+r)\right.\right. \\
\left.\left.-\frac{1}{3 r^{2}}\left((\alpha-n r)^{3}+r^{3}\right)\right]\right\} .
\end{gathered}
$$




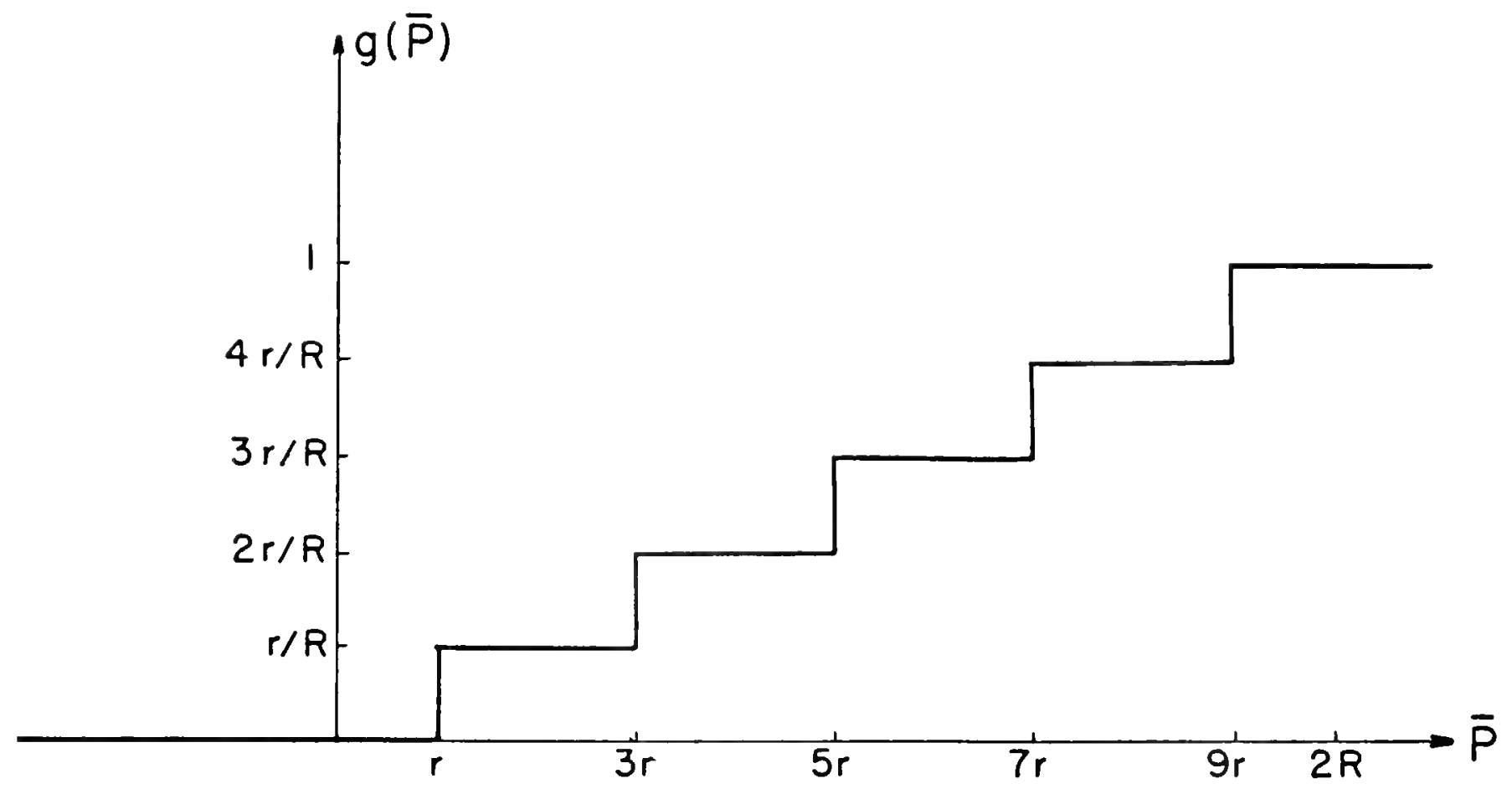

Figure 2. Definition of $g(\bar{P})$ for $R=5 r$. The definition may be extended in an obvious way for any mutiple of $r$.

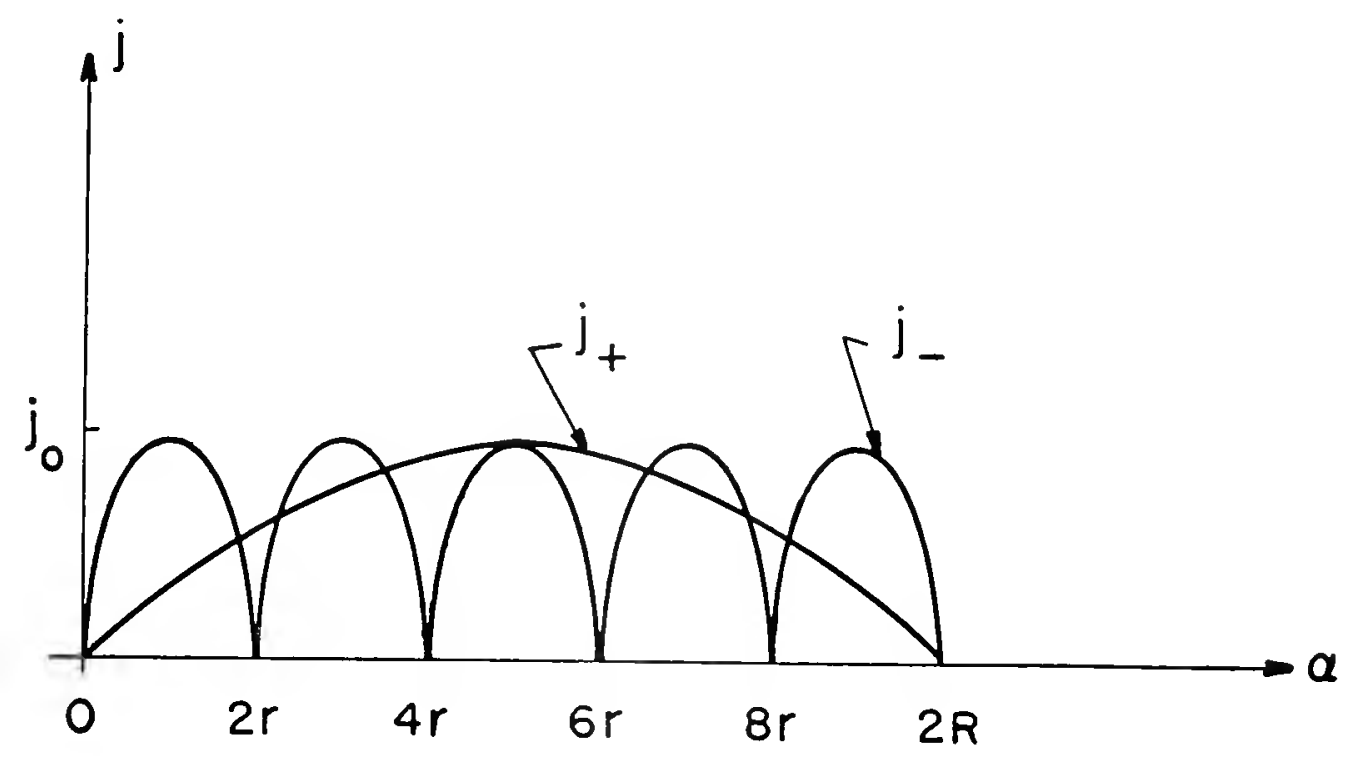

Figure 3. Ion and electron currents for $R=5 r$. 
This is essentially an equation of pressure balance. Grad ${ }^{3}$ has shown that

$$
p_{x y}^{(x)}= \pm \int_{0}^{x} i_{ \pm}(i) d x
$$

$( \pm)$

where $p_{x}$ is the indicated component of the ion or electron atress tensor and defined by the relation

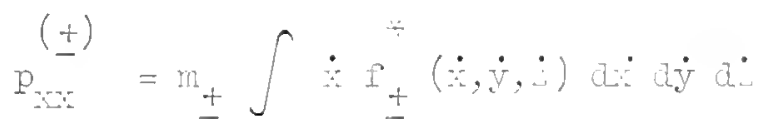

$$
\begin{aligned}
& =\frac{2}{m_{ \pm}^{2}} \int \sqrt{2 m+E-(p+e A / c)^{2}-Q^{2}} I_{ \pm}(E, F, Q) d E d F d Q .
\end{aligned}
$$

Equation (13) follows immediately on integration by parts with respect to $\alpha$. We see then that (12) may be written in the form

$$
B_{0}^{2} / 8 \pi=B^{2} / 8 \pi+p_{w}^{+}+p_{x}^{-}
$$

which states that the total pressure is a constant throughout the plasma and equal to its value at the interface. Note that the particle pressure at the interface, $\mathrm{p}_{\mathrm{x} x}^{( \pm)}(0)$ is zero.

In the problem considered here the ion and electron pressures are

$$
\begin{gathered}
p_{x x}^{+}=\frac{j_{0}}{e} R\left[\left(\frac{\alpha}{R}\right)^{2}-\frac{1}{3}\left(\frac{\alpha}{R}\right)^{3}\right] \\
p_{x x}^{-}=\frac{j_{0}}{e r^{2}}\left[2 / 3(n-1) r^{3}+r^{2}(\alpha-n r+r)-\frac{1}{3}\left((\alpha-n r)^{3}+r^{3}\right)\right] .
\end{gathered}
$$

Here again $\mathrm{n}$ is defined by the inequality (8). 
It is worthwhile digressing for a moment to note that all quantities vary only within the range $0<\alpha<2 R$, and are constant outside (see (1), (P) and (1)). From (11) it follows that a $(=e A(x) / c)$ is finite for all finite $x$, and therefore that the transition layer is finite. Furthermore one may give the following physical interpretation to the transition layer thiclness in terms of the particle trajectories. Returning to equations (3), which relate the particle trajectory to the constants of motion, it is easily seen that the ion which just reaches the plasma-vacuum interiace $\left(P=\sqrt{2 m_{+} E_{+}}\right)$wi.th no component of velocity along the magnetic field $(Q=0)$ will range from $\alpha=0$ at the left face of the boundary to $\alpha=2 \sqrt{2 m+E_{+}}=2 R$ at the right face. Thus the boundary layer thiclness is characterized by the orbit of that ion with maximum radius of curvature at the plasma-vacuum interface (see figure (4)). Returning now to (12) and integrating a second time we obtain $:$ as a function of $\alpha$

$$
\begin{aligned}
& x(\alpha)=\frac{c}{e B} \int_{0}^{\alpha}\left\{1+\frac{3}{8} \beta\left\{(\alpha / R)^{2}-\frac{1}{3}(\alpha / R)^{3}\right.\right. \\
& \left.\left.\quad+1 / \operatorname{Rr}^{2}\left[\frac{2}{3}(n-1) r^{3}+r^{2}(\alpha-n r+r)-\frac{1}{3}\left((\alpha-n r)^{3}+r^{3}\right)\right]\right\}\right\}^{-\frac{1}{2}} d \alpha
\end{aligned}
$$

where

$$
\beta=\frac{B_{0}^{2}-B_{\infty}^{2}}{B_{0}^{2}}=64 \pi j_{0} R / 3 e B_{0}^{2} .
$$

In (16) we have used (12) evaluated at $\alpha=2 \mathrm{R}$. 


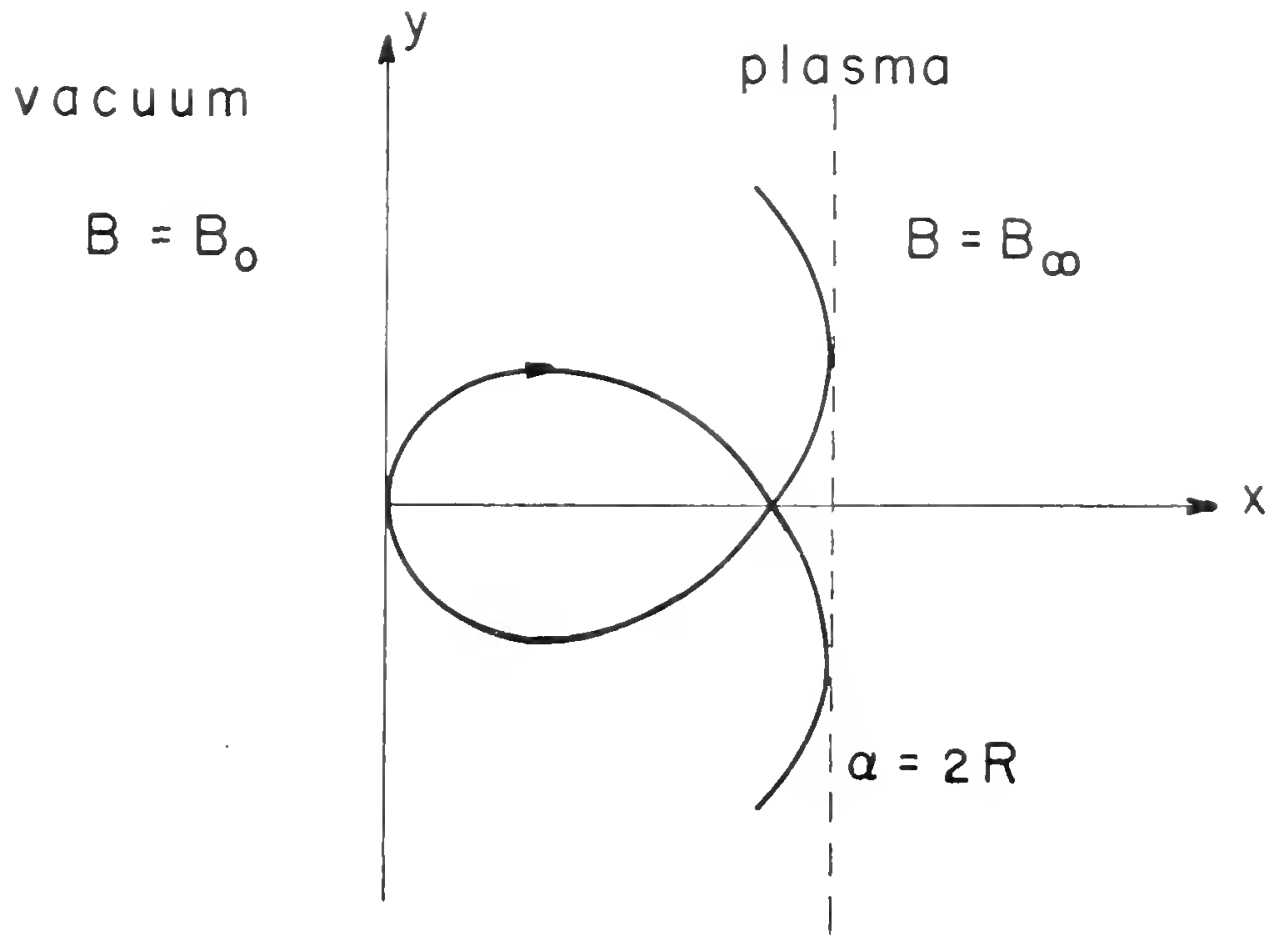

Figure 4. The edges of the transition layer bound the trajectory of the ion of maximum "diameter". 
Equation (15) together with (12), which may be written in the form

$$
\begin{aligned}
& B(\alpha)=B_{0}\left\{1+\frac{3}{8} B\left\{(\alpha / R)^{2}-\frac{1}{3}(\alpha / R)^{3}+1 / R r^{2}\right.\right. \\
& {\left.\left.\left[\frac{2}{3}(n-1) x^{3}+r^{2}(\alpha-n r+r)-\frac{1}{3}\left((\alpha-n)^{3}+x^{3}\right)\right]\right]\right\}, }
\end{aligned}
$$

determine the magnetic field parametrically as a function of $\mathrm{z}$. It mast be remembered in (17) that $\mathrm{n}$ is a discontinuous function of Although the integral in (15) is elliptic in form it is in general more practical to do the integration munerically. There is however a limiting case which proves to be of particular interest and for which an explicit solution is possible. We shall let the electron mass go to zero and the velocity go to infinity in such a way that the energy remains constant (and equal to the ion energy). In this limit we see that $x$ approaches zero and $n$ approaches infinity in such a way that the product $n r$ approaches $\alpha$ (see the inequality (II)). The electron pressure (equation (14)) becomes simply

$$
\mathrm{p}_{\mathrm{xx}}^{-}=2 / 3 \frac{\dot{j}_{0}}{\mathrm{e}} \mathrm{nr}=2 / 3 \frac{\dot{j}_{0}}{\mathrm{e}} \alpha
$$

The integral (15) may now be performed explicitly by solving a cubic equation. For $\beta=\frac{1}{2}$ the solution is

$$
\begin{aligned}
& B=B_{0} I / 4 \sqrt{\xi^{3}-5 \xi+12} \\
& X=\frac{R c}{\mathrm{eB}_{0}} 2(22)^{-1 / 4}[F(81.80, \varphi)-F(8 I .80,66.27)] \\
& \xi=\sqrt{22} \tan ^{2} \varphi / 2-3
\end{aligned}
$$


where $F(h, \varphi)$ is an elliptic integral of the first kind. In fisure (5) we have plotted the magnetic field profile. It is noteworthy that the slope of the magnetic field is not zero at $\alpha=0$ and $\alpha=2 \mathrm{R}$ despite the ract that $j_{+}$and $j_{-}$are sero there for all finite $r$ at these points (see (I)). The slope therefore approsches its limit discontinuously as r approaches zero.

The transition layer thickness in a plasma for which $\mathrm{B}=\frac{1}{2}$, measured in units of the diameter of an Lon in a uniform field $B_{0}$. is 1.17535. There are two averages with which this result might be compared. The first is the average diameter of an ion orbit moving in uniform fields $B_{0}$ and $B_{\infty}$ and the second the aiameter of an ion moving in a uniforn field which is the average of $B_{0}$ and $B_{\infty}$. These are 1.207 and 1.172 respectively.

For comparison we also carried out the integration (12) numerically for a plasma for which $\beta=\frac{1}{2}$ and $R=43 r$. For a hydrogen plasma $R=\sqrt{\frac{m_{F}}{m_{e}}} r=42.8 r$. It is not possible to distinguish graphically between this case and the previous one were $x=0$. In fact the transition layer thickness here is 1.17530 and differs from the former result only in the sixth significant figure. This very close agreement becomes somewhat less surprising when one observes that if the integral (15) is expanded in a power series in $\mathrm{r} / \mathrm{R}$ the term proportional to $\mathrm{r} / \mathrm{R}$ vanishes and the difference between the two results must be at most of order $(r / R)^{2}$. 


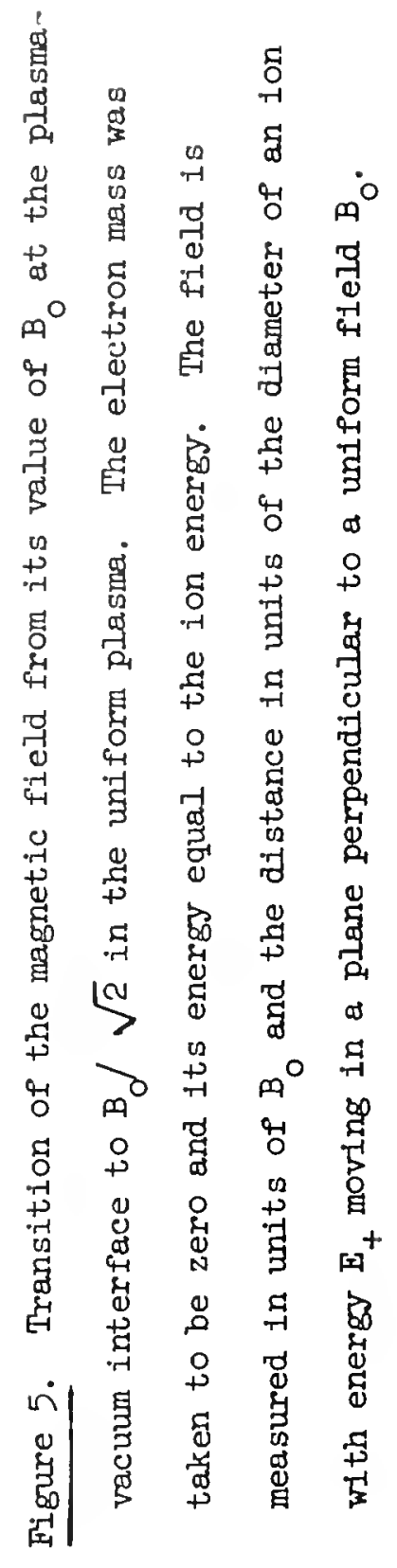


It shoula be recalled that the slope of the magnetic fiela must be zero at both ends of the transtion layer for a plasma with finite $r / R$. That this is the case for our simalated hydrogen plasma is not apparent from figure (5). It was not possible to indicate on the graph the very rapid fluctuations in the slope of the magnetic field (see (1) and figure (3) with $R=43 r$ ). The slope varies from cero to a maximum and back to sero again in a distance which is roughy l/43'rd of the transition layer thichness. The average slope over this range is that indicated in the figure.

\section{Guiding Center Approximation}

We have seen that the agreement between the "exact" results and the results obtained by setting the electron mass equal to zero is surprisingly good. It would be useful therefore to be able to obtain this linit without solving the integral equation ( 9 ), for if the ion distribution function were more complicated this equation might be very difficult. We shall avoid this intermediate step of determining the electron distribution function by showing that if the distribution function is factorable the electron pressure is directly proportional to the number density in the limit of zero mass. Since the density is a known function of $\alpha$ we may proceed immediately to solve the field equation (I).

To show this we return to the definitions of the pressure and number density 


$$
\begin{aligned}
& P_{X X}(\alpha)=\frac{2}{m^{3}} \int f(E, P, Q) \sqrt{2 m E-(P-\alpha)^{2}-Q^{2}} d E d P d Q \\
& N(\alpha)=\frac{2}{m^{2}} \int f(E, P, Q) / \sqrt{2 m E-(P-\alpha)^{2}-Q^{2}} d E d P d Q
\end{aligned}
$$

and consider the limit as $m$ approaches zero. The range of integration is over those values of $E, P$ and $Q$ for which the square root is real. We observe that as $m$ approaches zero the range of values that may be taken on by $P$ and $Q$ approaches zero. If we assume that the distribution function may be factored so that $f(E, P, Q)=f_{I}(P) f_{2}(E, Q)$ and if $f_{I}$ is a contimuous ${ }^{12}$ function of $P$ then we may evaluate $f_{I}$ inside the integrals at $\mathrm{P}=\alpha$ since in this limited range of integration the difference between $\mathrm{P}$ and $\alpha$ is proportional to $\sqrt{\mathrm{m}}$. We then have for the ratio of pressure and density

$$
\frac{p_{X X}(\alpha)}{N(\alpha)}=\frac{\frac{1}{m} \int f_{2}(E, Q)\left(2 m E-Q^{2}\right) d E d Q}{\int f_{2}(E, Q) d E d Q}
$$

where the right hand side is a constant with the dimensions of energy and hence proportional to the average energy per particle which we shall assume remains finite as $m$ approaches zero. It is in fact the average energy per particle associated with motion perpendicular to the magnetic field.

If we take $f_{2} \sim \delta\left(E-E_{0}\right)$ we obtain the results of the previous section (see equations (9) and (18)), namely

12 In the previous example $f_{l}(P)$ is not continuous, but approaches a continuous function as $m$ approaches zero. 


$$
p_{X:}=2 / 3 E_{0} I I
$$

or

$$
p_{x+t}=2 / 3 \frac{\dot{j}_{0}}{\mathrm{e}} \alpha
$$

If the plasma were Maxwellian, $f_{2} \sim e^{-E / k_{2} T}$, we find

$$
p_{X x}=\mathbb{N} \mathrm{KT}
$$

which is the equation of state of an ideal gas. As a lazt example let $f_{2} \sim s\left(E-E_{0}\right) s(Q)$ so that the particles are confined to move in a plane perpendicular to the macnetic field. The equation of state becomes

$$
p_{y x}=E_{0} N
$$

This last result was obtained previously by Tonts ${ }^{13}$ although in a somewhat different form. He analyoes in detail the motion of the particles in the guiding center approximation. It is not surprising that these two methods should give the same results, for letting the mass approach zero is equivalent to letting the Lamor radius approach zero, or in effect assuming that the Larmor radius is small in comparison to the distance within which the magnetic field changes by a significant fraction of itself. This is just the basis of the guiding center theory.

There is nothing in the preceeding analysis that restricts the results to either ions or electrons. In the transition layer problem,

13 I. Tonks, Phys. Rev. 97, 1443 (1955). See also L. Spitzer, Ir., Astrophys. J. 116, 299 (1952). 
however, the entire magnetic field variation takes place within one ion Iarmor diameter and so the guiding center approximation is not suitable. The electron on the other hand has 43 diameters in the layer.

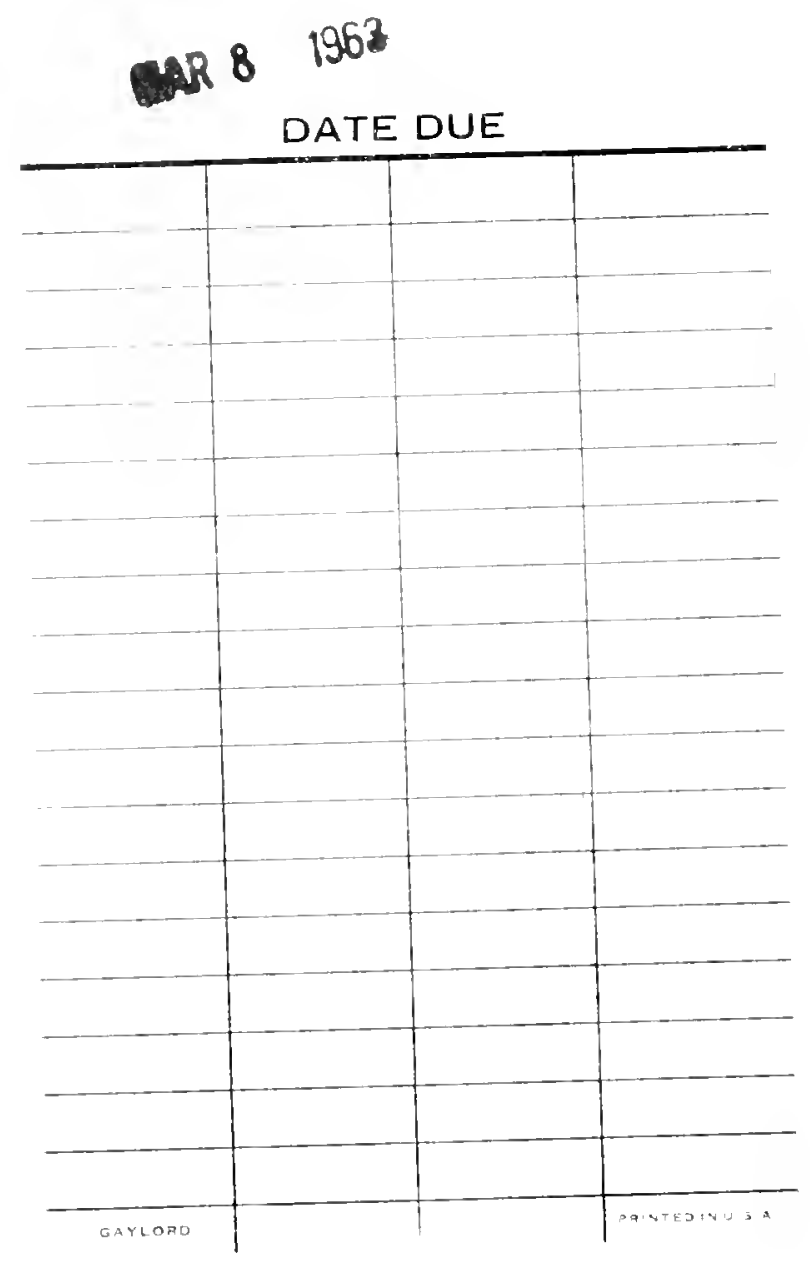




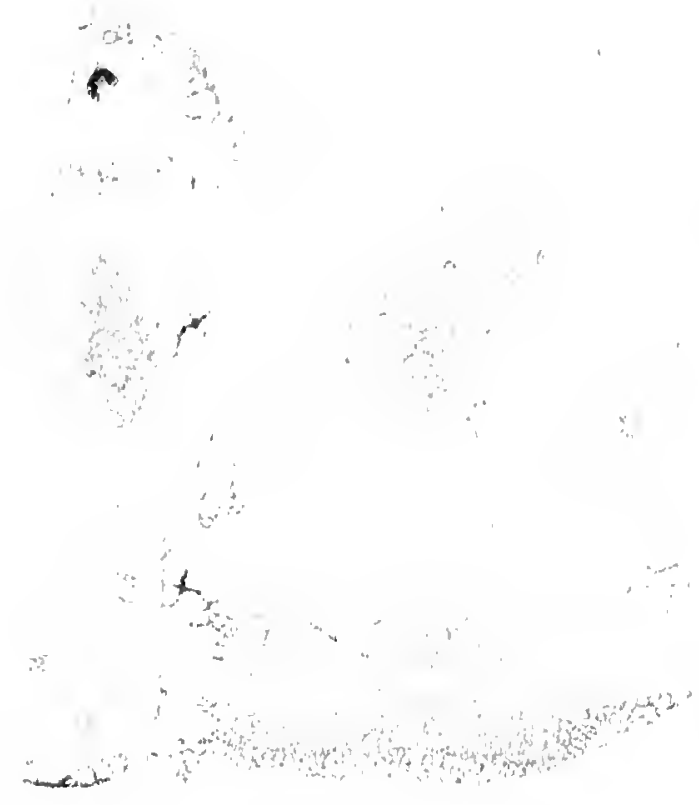


